\title{
Programa de escuela de padres para mejorar las relaciones afectivas en la familia y en la escuela, marzo 2013.
}

Parents School Program To Improve Loving Relationships In The Family And At School, In March 2013.

${ }^{1}$ Ysidoro Alejandría A. ${ }^{a}$ y ${ }^{2}$ Guillermo Zapata Ch. ${ }^{a}$

\section{RESUMEN}

Determina los efectos de la aplicación del programa de escuela para padres en las relaciones afectivas de los estudiantes. Por ello, se aplicó un pre test para medir las relaciones afectivas de los estudiantes en la dimensión familiar y escolar para luego desarrollar un programa con los padres y luego se aplicó un post test para medir los efectos producidos. La investigación fue de tipo experimental, con una población de 12 estudiantes quienes también conformaron la muestra. Se utilizó el diseño pre experimental con pre y post test. Los métodos de investigación utilizados han sido el inductivo-deductivo y analítico sintético. Y para el análisis de los resultados, se ha utilizado la estadística descriptiva en base a lo cual se presentan los resultados en tablas. Las relaciones afectivas de los estudiantes en el pre test, fueron: el 81, 3\% en nivel medio y $18,7 \%$ nivel alto. Luego de la aplicación del programa a los padres, el nivel medio es de $6,3 \%$ y el nivel alto es de $93,7 \%$. Los resultados nos muestran una diferencia significativa que nos conduce a concluir que el Programa Escuela para Padres, tiene efectos significativos en las relaciones afectivas de los estudiantes.

Palabras clave: Relaciones afectivas, familia, escuela y escuela para padres.

\section{ABSTRACT}

Determines the effects of the implementation of the School for Parents Affective Relations students. Therefore, a pre-test was applied to measure Affective relations students at home and school size and then develop a program with parents and then a post-test was applied to measure the effects produced. The research is experimental, with a population of 12 students who also formed the sample. The preexperimental design with pre and post test was used. The research methods used were the inductivedeductive and analytical synthetic. And to the analysis of the results, we used descriptive statistics based on which the results are presented in tables and graphs. Affective Relations students in the pretest, were: $81,3 \%$ on average, $18,7 \%$ high. After implementation of the program to parents, the average is $6,3 \%$ and the highest level is $93,7 \%$. The results show a significant difference that leads us to conclude that the School Parent Program, has significant effects on Affective Relations students.

Keywords: Affective relationships, Family, School, School for Parents

\footnotetext{
${ }^{1}$ Universidad Nacional de Jaén. Cajamarca, Perú

${ }^{2}$ Unidad de Gestión Educativa Local - Jaén, Cajamarca, Perú

${ }^{a}$ Licenciado en Filosofía y Ciencias Sociales
} 


\section{INTRODUCCIÓN}

En la Institución Educativa "Niño Jesús" del caserío La Unión, distrito de Sallique, provincia de Jaén, región Cajamarca, como en otras instituciones campesinas, los alumnos tienen inadecuadas relaciones afectivas en la familia (Pineda, 2011) y en la escuela. Y de una manera especial, en el segundo grado de educación secundaria, se ha detectado un alto grado de conflictividad expresada con malos comportamientos como continuas agresiones verbales, riñas, disputa por los espacios en el aula, se esconden las cosas, se muestran envidiosos, se registran robos de útiles, entre otros. Es decir, las relaciones afectivas son inadecuadas. Pero esto viene de la familia y el aula es el espacio donde hacen aflorar, su cólera, frustración, y descontento que traen de su entorno familiar (Fulquez, 2011).

La gran mayoría de padres de familia tienen escasos conocimientos de su papel de padres, la mayoría entiende que este papel, se limita en brindarle vestido, alimento, salud y educación (Wong y Yataco, 2012). La gran mayoría descuida el lado afectivo, en muchos casos por una errónea concepción y machismo.

Considerando que la familia es el primer núcleo de socialización y afectividad de una persona (Maestre, 2009). Se requiere de especial atención para potenciarla y que ejerza un efecto positivo en sus miembros, sobre todo en los hijos. Las actividades desarrolladas en el seno familiar tienen gran importancia en la formación de la personalidad y el éxito en el desarrollo del adolescente, de lo contrario el estudiante recibirá una formación negativa.
Existen, además, formas erróneas de crianza, que los padres deben reflexionar para ayudar en construir un ser individuo más humano. Así mismo, la investigación, teóricamente permitió establecer la relación directa que tiene la formación de los padres en la afectividad de los hijos (Sallés, 2012). Y en lo práctico, permitió establecer pautas y estrategias para mejorar las relaciones afectivas de los adolescentes tanto en la familia como en la Institución Educativa Niño Jesús de Sallique. Por otro lado, ayudó a los docentes, no solo a conocer la influencia de la escuela para padres, sino a poder aplicarlo pues quedó el programa en la Institución Educativa. Y a nivel de las familias, permitió a los padres de familia conocer su realidad y asumir con responsabilidad su compromiso en la educación de sus hijos para mejorar las relaciones afectivas (Psicodiagnosis.es, 2010).

La investigación tuvo su sustento en la teoría de las inteligencias múltiples de Gardner, pues nos presenta una comprensión amplia del ser humano y de las distintas formas que tiene para aprender y manifestar sus conocimientos intelectuales y sociales (Fumertón, 2011). De esta teoría se ha tomado sobre todo los aspectos relacionados a la inteligencia interpersonal (Ramón y García, 2010). Otra teoría que sustenta la investigación es la teoría de la inteligencia emocional de Goleman que recoge las inteligencias interpersonal e intrapersonal propuestas por Gardner, así como la teoría sociocultural de Vigotsky quien afirma que el contexto social influye en el aprendizaje más que las actitudes y las creencias, pues moldea los procesos cognitivos(García, 2011). 
En la investigación se asumen las relaciones afectivas como un conjunto de interacciones entre individuos en los que se crean vínculos y se establecen relaciones de intercambio y afecto y se aprende a resolver conflictos interpersonales armonizando los propios derechos con los derechos de los demás, en busca del bien común. Estas relaciones afectivas se manifiestan en la escuela y en la familia.

Las relaciones afectivas en la familia son relaciones intrafamiliares que incluye la percepción que se tiene del grado de unión familiar, del estilo de la familia para afrontar problemas, para expresar emociones, manejar las reglas de convivencia y adaptarse a las situaciones de cambio (Martínez y Pérez, 2012). Se manifiesta en tres dimensiones (Rivera y Andrade, 2010): 1) En la unión y apoyo al realizar actividades, convivir y apoyarse. Se asocia con un sentido de solidaridad y de pertenencia con el sistema familiar. 2) En la solución de las dificultades o el conflicto y la resolución del mismo. 3) En la expresión de la afectividad, es decir en la posibilidad de comunicar verbalmente las emociones, ideas y acontecimientos de los miembros de la familia dentro de un ambiente de respeto (López, 2011).

Las relaciones afectivas en la escuela son las muestras de solidaridad y participación, de ayuda mutua, de compañerismo, de respeto y valoración (Fingermann, 2011). Se manifiesta en la escucha activa a los compañeros. Una escucha profunda donde el receptor se adecua al tono anímico del que habla (ironía, humor, seriedad, preocupación (Lazo, 2012). Se manifiesta en el compañerismo al trabajar en equipo para generar un ambiente fraterno y participativo en el que la comunicación horizontal entre todos los integrantes favorezca el desarrollo de la imaginación creativa reflexiva; permita expresar lo que suponen acerca de la realidad, con toda la emotividad que el sujeto y las relaciones interpersonales implican y profundizan en el conocimiento y comprensión, y que posibilite identificar y solucionar las confrontaciones en equipo (Castellanos, 2011).

La mejora de estas relaciones afectivas tanto en la escuela y en la familia, se pueden abordar desde un programa que es una experiencia de aprendizaje planificada, estructurada, diseñada a sátisfacer las necesidades de los participantes (Unicef, 2011). Por tanto, el programa de escuela para padres es una estrategia con la finalidad de educar, orientar y capacitar a padres de familia, para fomentar su participación en la responsabilidad de guiar adecuadamente la formación de sus hijos, y a los estudiantes en el proceso de enseñanza-aprendizaje y en su desenvolvimiento para el desarrollo social, es decir, de su afectividad (ITACE, 2011).

\section{MATERIALES Y MÉTODOS}

La investigación tuvo su sustento en la teoría de las inteligencias múltiples de Gardner, pues nos presenta una comprensión amplia del ser humano y de las distintas formas que tiene para aprender y manifestar sus conocimientos.

La investigación fue experimental/causal explicativa, del tipo pre experimental con un solo grupo, por ello se utilizó el diseño pre test y post test. La población la conformaron los estudiantes de educación secundaria, pero la muestra se tomó intencionalmente a criterio del 
investigador, a 16 estudiantes del $2^{\circ} \mathrm{de}$ secundaria de la Institución Educativa "Niño Jesús” del caserío La Unión, distrito de Sallique, provincia de Jaén.

Los métodos de investigación utilizados fueron el deductivo-inductivo, combinando el trabajo de campo con la teoría para llegar a conclusiones. Así mismo, se estudiaron los hechos, partiendo de la descomposición del objeto de estudio que son las relaciones afectivas de los estudiantes, en sus dimensiones e indicadores, para estudiarlas en forma individual y luego de forma holística e integral.

La técnica utilizada fue la encuesta, cuyo instrumento fue un cuestionario aplicado a los 16 estudiantes de la muestra en dos momentos. Como pre test antes de aplicar el programa a los padres y luego como post test, es decir, después de desarrollar el programa de escuela de padres.

El instrumento antes de su utilización, fue sometido a juicio de expertos y a una prueba de confiabilidad, para lo cual se utilizó el coeficiente Alpha de Cronbach, que arrojó un puntaje global de 0,907, (con 0,902 para las relaciones afectivas familiares y 0,833 para las relaciones afectivas en la escuela), lo que determinó un alto grado de confiabilidad.

Así mismo, para el análisis de los datos se utilizó el software estadístico SPSS 22 para Windows, Microsoft Office Word y Excel 2013 para realizar algunas tablas, figuras y cálculos adicionales como para la redacción de la investigación. También se utilizó la estadística descriptiva y las medidas de tendencia central, así mismo, se utilizó la prueba de normalidad de Shapiro - Wilk, la Prueba de los rangos con signo de Wilcoxon.

\section{RESULTADOS}

Tabla 1. Nivel de relaciones afectivas en la familia, de los estudiantes del segundo grado de educación secundaria de la Institución Educativa "Niño Jesús" de La Unión, distrito de Sallique Jaén, octubre 2012 - marzo 2013, según pre y post Test.

\begin{tabular}{ccccc}
\hline Nivel & \multicolumn{2}{c}{ Pre test } & \multicolumn{2}{c}{ Post test } \\
\cline { 2 - 5 } & fi & $\%$ & fi & $\%$ \\
\hline Bajo & 0 & 0 & 0 & 0 \\
\hline Medio & 13 & 81,3 & 0 & 0 \\
\hline Alto & 3 & 18,7 & 16 & 100 \\
\hline Total & 16 & 100 & 16 & 100 \\
\hline
\end{tabular}

Fuente: Cuestionario aplicado (24/10/2012) y $(16 / 12 / 2012)$

Tabla 2. Nivel de relaciones afectivas en la escuela, de los estudiantes del segundo grado de educación secundaria de la Institución Educativa "Niño Jesús" de La Unión, distrito de Sallique Jaén, octubre 2012 - marzo 2013, según pre y post test.

\begin{tabular}{ccccc}
\hline Nivel & \multicolumn{2}{c}{ Pre test } & \multicolumn{2}{c}{ Post test } \\
\cline { 2 - 5 } & fi & $\%$ & fi & $\%$ \\
\hline Bajo & 0 & 0 & 0 & 0 \\
\hline Medio & 14 & 87,5 & 1 & 6,3 \\
\hline Alto & 2 & 12,5 & 15 & 93,7 \\
\hline Total & 16 & 100 & 16 & 100 \\
\hline
\end{tabular}

Fucntc: Cucstionario aplicado (24/10/2012) y $(16 / 12 / 2012)$

Tabla 3. Nivel global de las relaciones afectivas de los estudiantes del segundo grado de educación secundaria de la Institución Educativa "Niño Jesús" de La Unión, distrito de Sallique Jaén, octubre 2012 - marzo 2013, según pre y post test. 


\begin{tabular}{ccccc}
\hline Nivel & \multicolumn{2}{c}{ Pre test } & \multicolumn{2}{c}{ Post test } \\
\cline { 2 - 5 } & fi & $\%$ & fi & $\%$ \\
\hline Bajo & 0 & 0 & 0 & 0 \\
\hline Medio & 13 & 81,3 & 1 & 6,3 \\
\hline Alto & 3 & 18,7 & 15 & 93,7 \\
\hline Total & 16 & 100 & 16 & 100
\end{tabular}

Fuente: Cuestionario aplicado (24/10/2012) y $(16 / 12 / 2012)$

Los resultados obtenidos en el pre test nos reveló que las relaciones afectivas de los estudiantes se encuentran el 81,3\% en un nivel medio. Es importante tener en cuenta aquí, que no existen estudiantes con nivel bajo. Luego se desarrolló el programa escuela para padres, con los padres de familia de los estudiantes de la muestra. Luego, los resultados obtenidos después de la aplicación del programa escuela para padres, nos muestran una mejora significativa. En la dimensión relaciones afectivas familiares el $6,3 \%$ se encuentran en el nivel medio y sube al nivel alto con un $93,8 \%$. Y en la dimensión relaciones afectivas en la escuela, el 100\% de los estudiantes alcanzan el nivel alto.

Los estadísticos descriptivos nos mostraron que en las relaciones afectivas en la familia existe una variabilidad media de 3,2 en el pre test y 3,9 para el post test, con una desviación típica de 0,41 y 0,25 . Por su parte, en las relaciones afectivas en la escuela, la media del pre test es de 3,2 que se modificó en el post test con una media de 4 y una desviación típica de 0,00 . Por tanto y de acuerdo a la media global se observa que el 3,1 para el pre test y el 3,9 en el post test, son los que determinan la influencia del programa de escuela para padres aplicado.
La comparación de las medias después de la aplicación del programa a través de la prueba de los rangos con signo de Wilcoxon, nos reveló que los valores de significación $\mathrm{p}$ son menores a $0.05(0,000311)$ demostrando que la diferencia entre el pre y post test es muy significativa, con un $95 \%$ de confianza.

\section{DISCUSIÓN}

Los resultados obtenidos luego de la sistematización de los instrumentos aplicados a la muestra del estudio, diagnosticó la relación que existe entre la variable independiente (Programa de escuela para padres) y la variable dependiente (relaciones afectivas de los estudiantes), en el contexto del objetivo general planteado: determinar los efectos de la aplicación del programa escuela para padres, en las relaciones afectivas de los estudiantes de segundo grado de educación secundaria de la Institución Educativa "Niño Jesús" del caserío La Unión, distrito de Sallique, provincia de Jaén, octubre 2012 - abril 2013. En consonancia, la escuela para padres es un recurso de apoyo, que pretende coadyuvar a dar solución para mejorar la intercomunicación en el contexto familiar, es un espacio de reflexión y apropiamiento de estrategias educativas y conocimientos que les permite realizar una tarea educativa acorde al momento de cambio que viven sus hijos en la sociedad(Psicodiagnosis.es, 2010).

Con los resultados descritos anteriormente se puede afirmar que los efectos del programa han sido muy positivos. Por tanto, la familia es determinante en el comportamiento del niño y lo más importante es el hecho de que la familia es susceptible de aceptar un agente modificador 
que pueda inducirle a cambios orientados a buscar el logro personal del niño impedido. Hecho que es fortalecido por el programa de escuela para padres respecto a las inteligencias múltiples de las que nos habla. Y que en los talleres se evidencia en gran medida el nivel de influencia de los padres en el rendimiento académico de sus hijos (Wong y Yataco, 2012).

El pre test nos reveló en la dimensión relaciones afectivas familiares que los estudiantes obtuvieron un nivel medio entre 80 y $90 \%$. Es importante tener en cuenta que no existen estudiantes con nivel bajo en esta dimensión. Por lo que es de vital importancia la presencia continua y opinante de los padres, de diversas formas y sobre todo mejorando las relaciones afectivas (Psicodiagnosis.es, 2010). Se trata de mejorar la inteligencia interpersonal de los estudiantes cuyas relaciones afectivas se encuentran mayoritariamente $(80 \%)$ en el nivel medio.

Es importante tener en cuenta aquí, que no existen estudiantes con nivel bajo. Es un referente, relacionado con la influcncia del entorno en las características y aprendizaje de la persona (Ramón y García, 2010). En los talleres los padres adquirieron actitudes que favorecieron un ejercicio eficaz de la paternidad como comprender los estados emocionales de los hijos, expresarles su amor y establecer límites claros(Pineda, 2011).

Los resultados obtenidos después de la aplicación del programa escuela para padres, nos muestran una mejora en las dos dimensiones. En la dimensión relaciones afectivas familiares el 6,3\% se encuentran en el nivel medio y sube al nivel alto con un $93.8 \%$.
Esto indica que es en la familia donde se gesta el primer contacto emocional del ser humano, y por tanto, el contexto social influye en el aprendizaje más que las actitudes y las creencias, pues moldea los procesos cognitivos (García, 2011). $\mathrm{Y}$ en la dimensión relaciones afectivas en la escuela, el 100\% de los estudiantes alcanzan el nivel alto. Si asumimos que la afectividad es el conjunto de sentimientos expresados a través de acciones entre personas de cualquier contexto social en que están inmersos los individuos (Maioma, 2010). Es la escuela ese segundo espacio de interacción donde se desarrolla el proceso afectivo.

Los estadísticos descriptivos por su parte, nos muestran que en las relaciones afectivas en la familia existe una variabilidad media de 3,2 en el pre test y 3,9 para el post test, con una desviación típica de 0,41 y 0,25. Por su parte, en las relaciones afectivas en la escuela, la media del pre test es de 3,2 que se modificó en el post test con una media de 4 y una desviación típica de 0,00 . Por tanto y de acuerdo a la media global se observa que el 3,1 para el pre test y el 3,9 en cl post test, son los que determinan la influencia del programa de escuela para padres aplicado. Esto corrobora las conclusiones de que el trato, el respeto y acompañamiento de los padres $\mathrm{y}$ madres de familia en el proceso educativo de los niños y niñas en la escuela repercuten en el aprendizaje y en su rendimiento escolar.

En conclusión, la familia se considera como la unidad social básica, donde el individuo se forma desde la niñez para que en su edad adulta se conduzca como una persona productiva para la sociedad donde se desarrolla (Gómez, 2011). 
Y la participación de los padres, juega un rol decisivo y determinante, cuanto mayor es su involucramiento en la escuela; y que los espacios creados para los padres de familia, fue de gran importancia e influyó positivamente en los hijos.

\section{CONCLUSIONES}

Las relaciones afectivas del niño en la familia, antes de la aplicación del Programa escuela para padres, eran del nivel medio $81.3 \%$ y 18,7 nivel alto, sin embargo, luego del desarrollo del programa, cl nivel medio bajó a $6,3 \%$ y cl nivel alto subió a $93.7 \%$.

El programa de escuela para padres fundamentado en los aportes de la teoría de las inteligencias múltiples de Gardner, y en la teoría de la inteligencia emocional de Goleman, desarrollado con los padres de familia, produce efectos positivos muy significativos en los estudiantes, pues mejoran las relaciones afectivas de los estudiantes en la familia y en la escuela.

\section{AGRADECIMIENTOS}

Al director, docentes, familias y estudiantes, de segundo grado de secundaria de la Institución Educativa Secundaria "Niño Jesús" de la Unión, distrito de Sallique-Jaén.

\section{REFERENCIAS BIBLIOGRÁFICAS}

Castellanos, Claudia. Asertividad. marzo de 2011http://es.scribd.com/doc/51601960/manual -asertividad\#scribd.

Fingermann, H. El compañerismo en la escuela: La Guía de Educación. 2011 . http://educación.1aguia2000.com/general/elcompanerismo-en-la-escuela\#ixzz2AEb3ArkC.
Fulquez, Sandra. La inteligencia emocional y el ajuste psicológico: un estudio transcultural. Marzo de 2011. http://www.tdx.cat/bitstream/ handle/10803/9284/Tesis_Sandra_Carina_ Fulquez_Castro_parte_2.pdf; jsessionid= 837E13DD0276652F31D 9344C4ACC6B1A. tdx 1 ? sequence $=2$.

Fumertón, Mayra. Junio de 2011. www.feandalucia.ccoo.es/docu/p5sd7866.pdf. García, Maricarmen. Teorías del aprendizaje. noviembre de 2011. http://teoriasohico.blogspot. com/2011/11/bruner-arriba-vigotsky-abajo.html.

Gómez, G. La clave de la educación e s 1 a a fectividad. 2011 . h t t p://m. vanguardia.com/vida -y-estilo/jovenes/104954-la-clave-de-laeducación-es-la-afectividad.

Gonzáles, E. 2005. Educar en la afectividad. http://www2.amigonianos.org/surgam/articulos /504/12\%20EDUCAR\%20EN\%20LA\%20 AFECTIVIDAD.pdf (último acceso: 06 de 12 de 2012).

Hernández, Roberto, Carlos Fernández, y Pilar Baptista. Metodología de la investigación. Chile: M c Graw hill, 2010 . ITACE. Escuela para Padres y Madres. México DF: Secretaría de Educación, 2011.

Lazo, Pablo. Habilidades sociales básicas en la relación con el / la cliente/a. octubre de 2012. http://www.raco.cat/index.php/ FducacioSocial/article/viewFile/250177/369142.

En la relación con el / la cliente/a. octubre de 2012. http://www.raco.cat/index.php/ EducacioSocial/article/viewFile/250177/369142. 
Unicef. Manual para el trabajo con las familias.

$\mathrm{Fe} \mathrm{bre} \mathrm{ro} \quad \mathrm{de} \quad 2011$.

h t t p : / / w w w. unicef.cl/web/w pcontent/uploads/doc_wp/Manual $\% 20$ para $\% 2$ 0el\%20Trabajo\%20con\%20Familias.pdf.

Wong, C, y Yataco P. «Administración del tiempo por parte de los padres de familia y su influencia en el rendimiento académico de los estudiantes de quinto de secundaria de la I.El "Melchorita Saravia" Grocio Prado-Chincha, 2011".» Tesis de maestría, Universidad César Vallejos, Trujillo, 2012.

\section{Correspondencia:}

Ysidoro Alejandría Alejandría

Calle San Luis No 716- Morro Solar - Jaén.

yalejandriaalejandria@unj.edu.pe 